\title{
Ballistic failure processes in alumina
}

\author{
D.G. Brandon, L. Baum and D. Sherman
}

Department of Materials Engineering, Technion-Israel Institute of Technology, Haifa 32000, Israel

\begin{abstract}
This is a preliminary report in a new program of research to evaluate the basic failure processes occurring in dense ceramics during projectile penetration, and, in particular, to study the rôle played by mechanical constraint in inhibiting some failure modes. In this contribution we describe a method for monitoring the response of selected ceramics to a highly localized pressure pulse under conditions of controlled constraint and distinguish the effect of constraint on the three primary failure modes:

1. Crushing, comminution and erosion in the zone of high compressive stress which exists near the contact surface between the projectile and the impacted ceramic.

2. Nucleation and propagation of a cone crack, predominantly in mode II (the shear stress mode) to form a ceramic plug ahead of the advancing projectile.

3. Fragmentation associated with radial (long-term) cracks nucleated and propagated by tensile stresses in the target material surrounding the contact zone.

Constraint acts primarily to prevent the formation of a cone crack, allowing higher compressive stresses to be reached in the contact zone. At the levels of constraint studied so far, radial (tensile) cracks always terminate the failure process, releasing the compressive stresses in the contact zone.
\end{abstract}

\section{INTRODUCTION}

Over the past two decades a wide range of monolithic ceramics [1-3] have been evaluated for their ballistic performance and the results have led to a well-established understanding of the requirements for ceramic armor. No one mechanism dominates dynamic failure during projectile penetration. Deflection of the projectile, erosion of the projectile and target [4-6], shock-wave propagation and reflection [7], crack initiation and coalescence [8-11], the rigidity of the armor module, the constraints introduced by the mounting system, and the design of the backing layer [4]: all play a rôle in determining the minimum weight of armor required to defeat a given threat.

Samples of alumina tiles subjected to planar impact in the pressure range up to and beyond the Hugoniot elastic limit (HEL: typically 6-10 GPa ) show a clear threshold for damage which depends on the grade of alumina [12]. Although some dislocations are introduced by impaction no appreciable plastic flow occurs at sub-HEL levels of loading. Microcracking initiates at 40 to $60 \%$ of the HEL [13], typically 2.5 to $4.5 \mathrm{GPa}$. The planar impact configuration corresponds to a fully constrained test, but with no initial stress on the target. The failure surface of spalled alumina 
samples has demonstrated that spall failure occurs by the accumulation of microcrack damage in the spall zone [14]. Fragmentation of a ceramic tile under projectile impact includes not only crushing processes, but also the dynamic attrition and erosion at the interface between the ceramic and the projectile, as well as radial, transverse and spall cracks which determines the size distribution of the fragments.

The damage resulting from standard projectile impact on a brittle target has been studied over a period of at least 20 years and the damage corresponds to the three stages:

1. Crushing occurs in the high pressure compressive zone in the region of contact. This zone comprises three transitions associated with the onset of micro-cracking, the formation of interconnected cracks and fragmentation (loss of ceramic continuity).

2. Dynamic attrition and erosion at the interface between the ceramic and the projectile proceeds by a process of high pressure grinding. The resultant mix of target and impactor particles is accelerated and ejected from the front surface of the target.

3. The network of radial, transverse and spall cracks spreading from the contact zone determines the size distribution of the ceramic fragments. It is these last, long-term failure processes which are expected to be most affected by constraint of the target.

The present work reports a test for the effect of confinement on the ballistic failure mechanisms in a ceramic. The test is aimed at evaluating both the effect of constraint and the interaction between the different dynamic failure processes which occur during penetration.

\section{TEST PROCEDURE}

A flat punch was indented into a ceramic tile in a low-velocity drop-weight experiment. The $5.4 \mathrm{kgm}$ weight was released from a height of 0.2 to $2 \mathrm{~m}$ and the terminal velocity and deceleration monitored on an oscilloscope. The testing conditions translate to a localized pressure pulse of 1 to $5 \mathrm{GPa}$ and a pulse duration of 1 to $2 \mathrm{~ms}$. The velocity of the impactor is typically $2-6 \mathrm{~ms}^{-1}$. The design of the specimen module is shown in Fig.1. The ceramic plate is mechanically clamped by four bolted steel bars. A torquemeter is used to control the moment applied to each bolt. The ceramic plate rests on a slightly undersize support block, and this specimen tile, together with the support plate and clamping bars, is sandwiched between two stainless steel plates which are in turn bolted to the assembled clamping bars. The impedance of the support block (steel, aluminium or polymethylmethacrylate - PMMA) determine the short-term release wave history in the contact zone. The total thickness of the ceramic tile and aluminum backing plate is slightly greater than the thickness of the clamping bars. The impactor is a $2 \mathrm{~mm}$ diameter tungsten carbide pin (Vickers Hardness 1650) mounted in a $6 \mathrm{~mm}$ diameter T15 tool steel plunger. The impactor pin has $1 \mathrm{~mm}$ of travel before the drop-weight load assembly comes in contact with the guide plate. To maintain this distance and ensure that the impactor pin surface is accurately parallel to the plane of the tile, a 1 $\mathrm{mm}$ thick disk is inserted during the mechanical finishing of the impactor assembly.

\section{PRELIMINARY RESULTS}

The parameters for the preliminary tests are given in Table 1. In the first test the ceramic was unconstrained and the load was released from a height of $1 \mathrm{~m}$. Failure was dominated by radial cracking, while the region beneath the impactor was crushed and a star shaped zone at the bottom surface of the tile was 'punched out' by the impact. In the second test the ceramic was constrained by the clamping bars, but the plunger was appreciably undersize. Furthermore, the plunger was of (low hardness) stainless steel rather than tool steel. Again the load was released from $1 \mathrm{~m}$. The maximum registered impact pressure was $5.06 \mathrm{GPa}$ and the first sign of failure (non-linearity of the pulse rise) was at $4.65 \mathrm{GPa}$. The width of the first pulse was $1.06 \mathrm{~ms}$. The impact zone was asymmetrically indented, but very little other damage was visible. The stainlesssteel plunger was barreledby the impact. 
Table 1

Parameters for drop-weight tests

$\begin{array}{cccccc}\text { Test \# } & \begin{array}{c}\text { Drop-weight } \\ \text { Height, } \mathrm{m}\end{array} & \begin{array}{c}\text { Velocity } \\ \mathrm{ms}^{-1}\end{array} & \begin{array}{c}\text { Pulse Height } \\ \text { GPa }\end{array} & \begin{array}{c}\text { Pulse Width } \\ \text { ms }\end{array} & \begin{array}{c}\text { Constraint } \\ \text { (Torque) }\end{array} \\ 1 & 1.00 & 4.34 & - & & \text { none } \\ 2 & 1.00 & 4.16 & 5.06 & 1.06 & 400 \\ 3 & 1.02 & 4.50 & 4.05 & 2.00 & 350 \\ 4 & 1.40 & 5.26 & 3.80 & 2.04 & 350\end{array}$

In the third test more attention was paid to the tolerances and the plunger material was replaced by the T15 tool steel finally selected. The maximum pulse pressure was $4.05 \mathrm{GPa}$ and the width of the first pulse was $2.00 \mathrm{~ms}$. A crush zone was visible beneath the impactor, together with through-thickness radial cracking. A very fine deposit of alumina powder was observed on the aluminum backing plate and replicated the geometry of the radial cracks. The implication is that eroded alumina powder, created in the contact zone, is transported throughout the crack system when radial cracking releases the compressive stress. The aluminum alloy backing plate was also observed to be extensively cracked immediately beneath the impact zone, although these cracks did not extend more that a few $\mathrm{mm}$ from the center of impact. For the fourth test the specimen module was identical to that used in the third test, but the drop-weight was now released from $1.4 \mathrm{~m}$. The observed peak pressure was actually less $(3.80 \mathrm{GPa})$ although the pulse width was slightly greater ( $2.04 \mathrm{~ms})$. The damage, however, was more extensive. The pulse records were extremely sensitive to the exact geometry of the specimen module. The compressive damage introduced by the drop-weight impact ought to be comparable to that under planar impact. The static compressive strength of dense, monolithic alumina is of the order of 3-4 GPa, although these values are seldom reached, since parasitic bend stresses in the load train usually initiate premature failure.

Ballistic damage development is a complex process, evolving in three dimensions as a function of time. In general, the majority of reported studies on ballistic damage refer to incident projectile impacts normal to a planar specimen surface. These geometries (which include the present studies) introduce an axis of damage symmetry parallel to the momentum vector of the impactor. In the Hopkinson bar test the free surface of the specimen, parallel to the direction of impact, allows radial relaxation to occur during the early stages of the event. In planar impact tests, on the otherhand, the specimen dimensions are such that initially no radial relaxation occurs. In both cases the ballistic damage and stress history of a volume element vary with the distance beneath the contact surface but are independent of the distance from the axis of symmetry. In the present test the kinetic energy stored in the impactor assembly is between 10 and $100 \mathrm{~kJ}$. For comparison, a 0.3 cal. steel round traveling at $800 \mathrm{~ms}^{-1}$ has a kinetic energy of about $1.25 \mathrm{MJ}$. On the otherhand the diameter of the impactor pin used in the present test is only $2 \mathrm{~mm}$, while the 0.3 cal. round has a diameter of $7.62 \mathrm{~mm}$. The energy to be dissipated per unit area of contact during projectile penetration is thus about $14 \mathrm{~kJ} . \mathrm{mm}^{-2}$ for the steel round and between 3 and $30 \mathrm{~kJ} . \mathrm{mm}^{-2}$ for the drop-weight impactor.

The damage resulting from the drop-weight impact was evaluated in four damage zones: the impactor pin and plunger, the top surface of the ceramic tile, the bottom surface of the ceramic tile and the support plate. In all tests the impactor pin was fragmented. Since the pin/plunger assembly is longer than the tile thickness, pin fragmentation should occur at relatively long times. At the lower impact velocity the tool steel plunger appeared undamaged, but at the higher impact velocity the plunger was cracked longitudinally in the region of the impactor pin while the pin itself was chipped. Without constraint, radial cracking of the tile was fully developed and a crushing zone was visible at the point of impact (Fig.2). In the second test on the constrained tile no radial cracking was detected, although there was some indentation of the specimen tile and a die penetrant 
(fuchsin) revealed a well-developed circumferential crack at the edge of the indented region. In the third test radial cracking was readily visible with the help of the dye penetrant and was essentially unbranched. Crushing and fragmentation in the impact zone was less developed than in the first test. In the final test the crushed zone appeared similar to that in the third test, but the radial crack system exhibited numerous bifurcations, associated with higher crack velocities due to the higher kinetic energy of the impactor.

The crack system visible at the bottom surface of the tile provides information on the propagation of damage through the tile thickness. In the first, unconstrained test a star-shaped plug was ejected beneath the contact zone. This plug is associated with the absence of lateral constraints which permitted circumferential cracking to propagate in shear through the thickness, separating this central plug from the remainder of the tile. The transition from the circular, circumferential cracking, observed at the top surface, and the star shape, observed at the bottom surface, is taken as indicative of the interaction between the circumferential crack system and the reflected shock waves from the free edges of the square tile during through-thickness shear crack propagation. No damage was found on the bottom surface of the tile from the second test, consistent with the absence of radial cracking on the top surface. Removal of the (aluminum) support plate from the third test tile revealed a radial pattern of fine white lines on the surface of the plate, which matched the radial crack pattern at the bottom surface of the tile. This deposit is alumina 'dust' ejected from the eroded zone beneath the impactor pin during the pressure release which occurs as a result of radial crack propagation. In this third test no 'plug' was formed and the radial cracking at the bottom surface of the tile initiated immediately beneath the central region of the contact zone (Fig.3). This is consistent with the effect of radial constraint in suppressing circumferential shear crack propagation. In the case of the fourth test, at the higher impact velocity, the crack pattern at the bottom surface was less bifurcated than the top surface pattern. The crack propagation direction for the radial cracks is not strictly radial, but rather through-thickness (as in a double torsion fracture toughness test). This could indicate an appreciable bend stress on the tile, due either to constraint asymmetries or to the higher impact velocity. No damage to the 6061 aluminum support plates was observed in the first two tests. However, both the tests undertaken with the tool steel plunger for the impactor assembly resulted in localized radial cracking of the aluminum plate immediately beneath the contact zone.

Fractographic examination in the scanning electron microscope (SEM) has been limited to the central plug which formed in the unconstrained specimen and the radial crack surfaces. Fig. 4 shows the plug geometry. The point of impact is at the bottom of the figure and partial protrusion of the plug at the rear surface of the target is clearly visible. Just over $1 \mathrm{~mm}$ of material has been lost from the crush zone during specimen preparation and mounting. The surface of the plug, which is the exposed circumferential crack surface, was very rough (Fig.5). The radial crack surfaces are much smoother than the plug surface, with evidence of multiple shock reverberations (Fig.6), of which the most prominent correspond to the limit of the crushed zone (the effective depth of projectile penetration).

\section{DISCUSSION \& CONCLUSIONS}

The present drop-weight simulation test could form the basis for a systematic quantitative evaluation of the nature of impact damage in various grades of ballistic ceramic. The kinetic energy density in the area of contact of the impactor pin $\left(3\right.$ to $\left.30 \mathrm{~kJ} \cdot \mathrm{mm}^{-2}\right)$ satisfactorily brackets that delivered by an armor piercing round, so that the results should be directly relevant to ballistic performance. The pulse duration in the present test, of the order of $1 \mathrm{~ms}$, is also comparable to the time scale over which primary events are expected to occur in a ballistic event. The damage sequence accompanying projectile penetration in the present test corresponds to three distinct stages: 
1. Crushing and erosion of the ceramic in the high pressure contact zone developed between the impactor pin and the target tile continues as long as the pressure in the contact zone exceeds the compressive strength of the ceramic.

2. The initiation of crushing damage is followed (independently) by the formation of a circumferential tensile crack around the contact zone which can propagate through the tile thickness to form a shear plug. In a constrained geometry the depth of propagation is limited.

3. Radial crack formation terminates the crushing and erosion processes by releasing the pressure due to the impact and the volume changes which accompany crushing damage. The tile 'bursts' when the pressure in the crushed zone exceeds the sum of the constraining pressure and the tensile strength of the ceramic.

Plug formation was prevented by moderate constaints, while long-term radial cracking was inhibited, but not prevented. The rough fracture surface of the shear plug, when compared to the smooth fracture surface of the coarse radial fragments, is assumed to reflect the much higher crack propagation resistance for the former process

\section{REFERENCES}

1. Brandon D.G. 'Ceramic Armor', Encyclopedia of Adv.Cer.Mat. (Pergamon 1991) pp.22

2. Laible R.C., 'Ballistic materials and penetration mechanics' ed. Laible.R.C. (Elsevier Sci.Publ.1980) pp.133

3. Wilkins M.L., Cline C.F. and Hondol C.A., Tech.Rep. UCRL-50694 (Lawrence Rad.Lab. 1969)

4. Wilkins M.L., 'Ballistic materials and penetration mechanics' ed. Laible.R.C. (Elsevier Sci.Publ.1980) pp.225

5. Ravid M., Bodner S.R. and Holcman I., 10th Int.Symp. on Ballistics, Calif. 1987

6. Ravid M. and Bodner S.R., Int.J.Eng.Sci. 21 (1983) pp.577

7. Kolsky H., 'Ballistic materials and penetration mechanics' ed. Laible.R.C. (Elsevier Sci.Publ.1980) pp.185

8. Lee Y.J., Int.J.Fract. 57 (1992) pp.25

9. Ma C.C. and Chen S.K., Int.J.Fract. 58 (1992) pp.345

10. Kobayashi A.S., Mat.Sci.Eng. A143 (1991) pp.111

11. Rosenberg Z., Yeshurun Y. and Brandon D.G., J. de Phys. C5 [8] (1985) pp.331

12. Clime C.F. and Wilkins M.L., Tech.Rep. UCRL-71455 (Lawrence Rad.Lab. 1969)

13. Rosenberg Z. and Yeshurun Y., J.Appl.Phys. 58 (1985) p.3077

14. Roylance M.E., Roylance D. and Sultan J., J.Am.Chem.Soc. (1976) p. 192

\section{ACKNOWLEDGEMENTS}

This work was supported by the Israel Ministry of Defence under Contract \#193/3944/9. The authors are grateful to Dr. Y.Gefen for his interest and encouragement and Dr. Y. Yeshurun for many stimulating discussions. The drop-weight tester used for these experiments was developed in the Mechanics Laboratory of the Faculty of Mechanical Engineering at the Technion. 


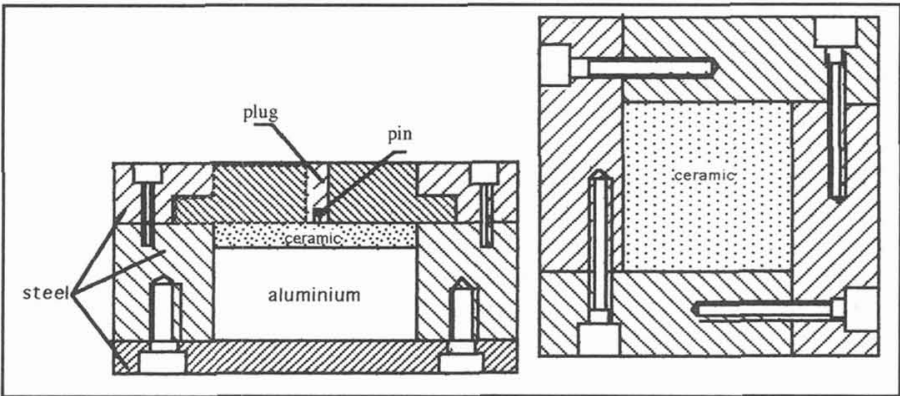

Fig. 1 Ceramic tile assembly with impactor pin in place (left) and plan of clamping geometry for the ceramic tile (right)

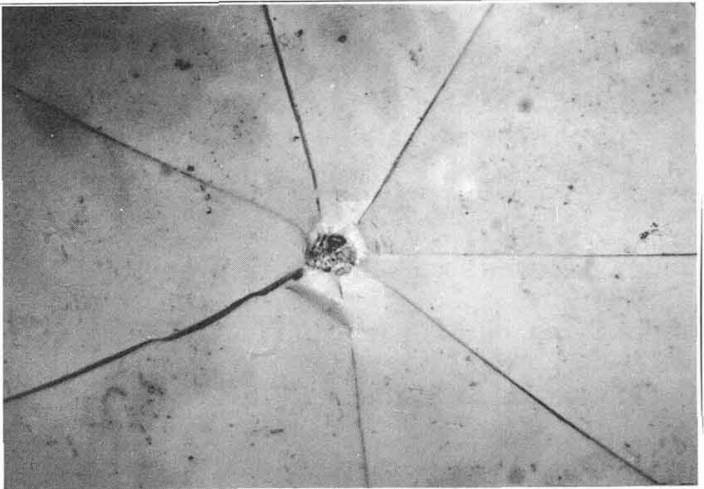

Fig. 2 Unconstrained specimen showing radial cracking and crushed impact zone

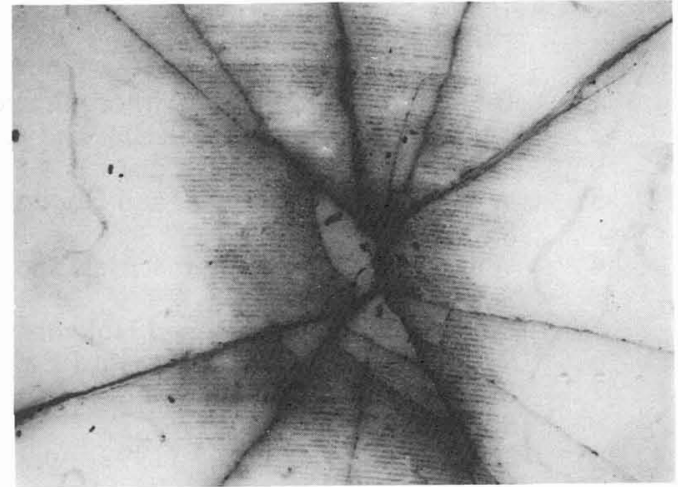

Fig. 3 Radial cracking at bottom surface of constrained low energy impact tile
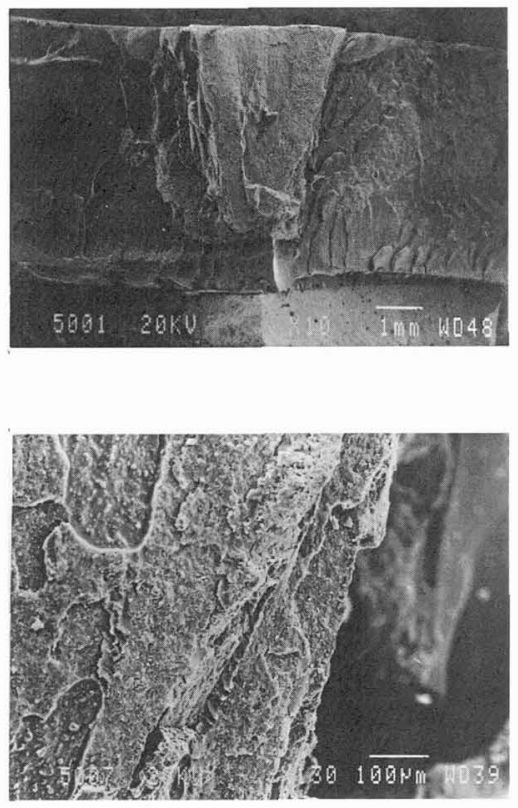

Fig. 4 SEM image of central region in an unconstrained tile. The plug formed by cone cracking is in the centre with the crush zone at the bottom. The tile thickness is $5 \mathrm{~mm}$. Smooth radial crack surfaces are to right and left.

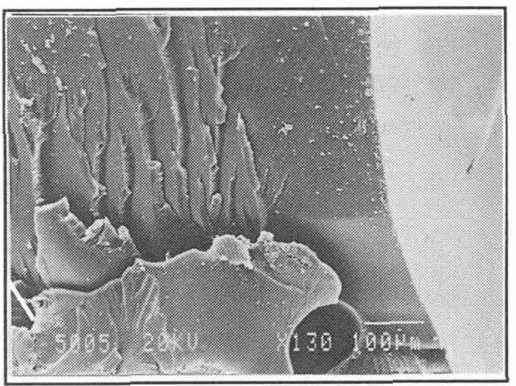

Fig. 6 Evidence for shock reverberations on smooth radial crack surface

Fig. 5 Fracture surface of 'plug' formed predominantly by Mode-II shear. 